\title{
Claude Debru - « Penser avec les mains » : critique génétique et épistémologie
}

Entretien avec Jean-Louis Lebrave

\section{Claude Debru et Jean-Louis Lebrave}

\section{OpenEdition}

\section{Journals}

\section{Édition électronique}

URL : https://journals.openedition.org/genesis/148

DOI : $10.4000 /$ genesis. 148

ISSN : 2268-1590

\section{Éditeur :}

Presses universitaires de Paris Sorbonne (PUPS), Société internationale de génétique artistique littéraire et scientifique (SIGALES)

\section{Édition imprimée}

Date de publication : 20 juin 2010

Pagination : 219-228

ISBN : 978-2-84050-697-3

ISSN : 1167-5101

\section{Référence électronique}

Claude Debru et Jean-Louis Lebrave, «Claude Debru - « Penser avec les mains » : critique génétique et épistémologie », Genesis [En ligne], 30 | 2010, mis en ligne le 17 mai 2013, consulté le 30 mars 2023. URL : http://journals.openedition.org/genesis/148; DOI : https://doi.org/10.4000/genesis.148 


\section{«Penser avec les mains": critique génétique et épistémologie Entretien avec Jean-Louis Lebrave \\ Claude Debru}

Ce numéro de Genesis aurait été incomplet sans une réflexion sur les archives scientifiques et l'apport de la critique génétique dans l'exploitation de celles-ci. Claude Debru, directeur du département de philosophie de l'École normale supérieure, membre correspondant de l'Académie des sciences, spécialiste de l' histoire des sciences du vivant, a bien voulu répondre à nos questions.

JeAn-Louis LeBrave - Dans le numéro de Genesis consacré à l'écriture scientifique1, Anouk Barberousse et Laurent Pinon mettent en avant l'écriture comme domaine d'intervention privilégié de la critique génétique en histoire des sciences. Qu'en pensez-vous?

CLAUDE DEBRU - Je n'ai jamais mieux appréhendé la dimension de créativité scientifique d'un chercheur qu'en consultant ses manuscrits, par exemple ceux de Claude Bernard, ou d'autres qu'il m'est arrivé de consulter dans telle ou telle occasion. Seuls les manuscrits permettent de saisir sur le vif le «style » de tel ou tel grand créateur scientifique. Dans le cas de Claude Bernard, dont nous reparlerons tout à l'heure, on y saisit sa merveilleuse capacité à varier ses expériences, ce que j'appellerai son imagination combinatoire. Ce qu'on observe également, c'est que l'écriture est inséparable du dessin. On voit que Claude Bernard pense par images : il accompagne ses projets d'expériences conceptualisées de dessins très détaillés. J'ai par exemple sous les yeux une grenouille décérébrée dont il ne reste quasiment plus que les deux jambes et la colonne vertébrale (voir fig. 1). Le dessin montre comment, à l'aide de pinces électriques, Claude Bernard cherche à stimuler telle ou telle partie de son corps. Les trajets nerveux ascendants ou descendants sont très bien définis. On voit à quel point l'imagination du physiologiste est inséparable de la création d'images visuelles, c'est-à-dire de visualisations des expériences dans lesquelles la dimension anatomo-fonctionnelle est parfaitement rendue. Cet aspect des manuscrits est à mon sens absolument irremplaçable. J'ajouterai qu'on y voit aussi comment Claude Bernard laisse libre cours à son imagination dans d'autres domaines que le domaine purement scientifique, puisqu'il se délasse en faisant des portraits.

J.-L. L. - Vous venez d'évoquer une dimension très importante pour la critique génétique : les cahiers de laboratoire contiennent beaucoup plus que les seules données textuelles. De même que les manuscrits littéraires portent la trace du processus de création de l'écrivain, peut-on dire d'une manière analogue que les cahiers de laboratoire contiennent les traces du chercheur au travail?

C. D. - Ce point est excessivement important. Pourquoi ? Parce qu'un chercheur ne s'arrête jamais. Il pense trouver une solution pour le problème qu'il explore, et cette solution fait bien évidemment émerger de nouveaux problèmes. Dans le cas de Claude Bernard, on peut voir qu'il est arrivé à des solutions provisoires, avec parfois des régressions dans la recherche ultérieure. C'est donc un travail incessant. Cette remarque est particulièrement vraie pour la physiologie expérimentale à ses débuts, c'est-à-dire à un stade de la science où l'inconnu prédomine largement sur le connu. Le connu, c'est Claude Bernard qui, en progressant, l'établit peu à peu.

1. Anouk Barberousse et Laurent Pinon (dir.), Genesis, n² 20, «Écriture scientifique », Paris, 2003. 


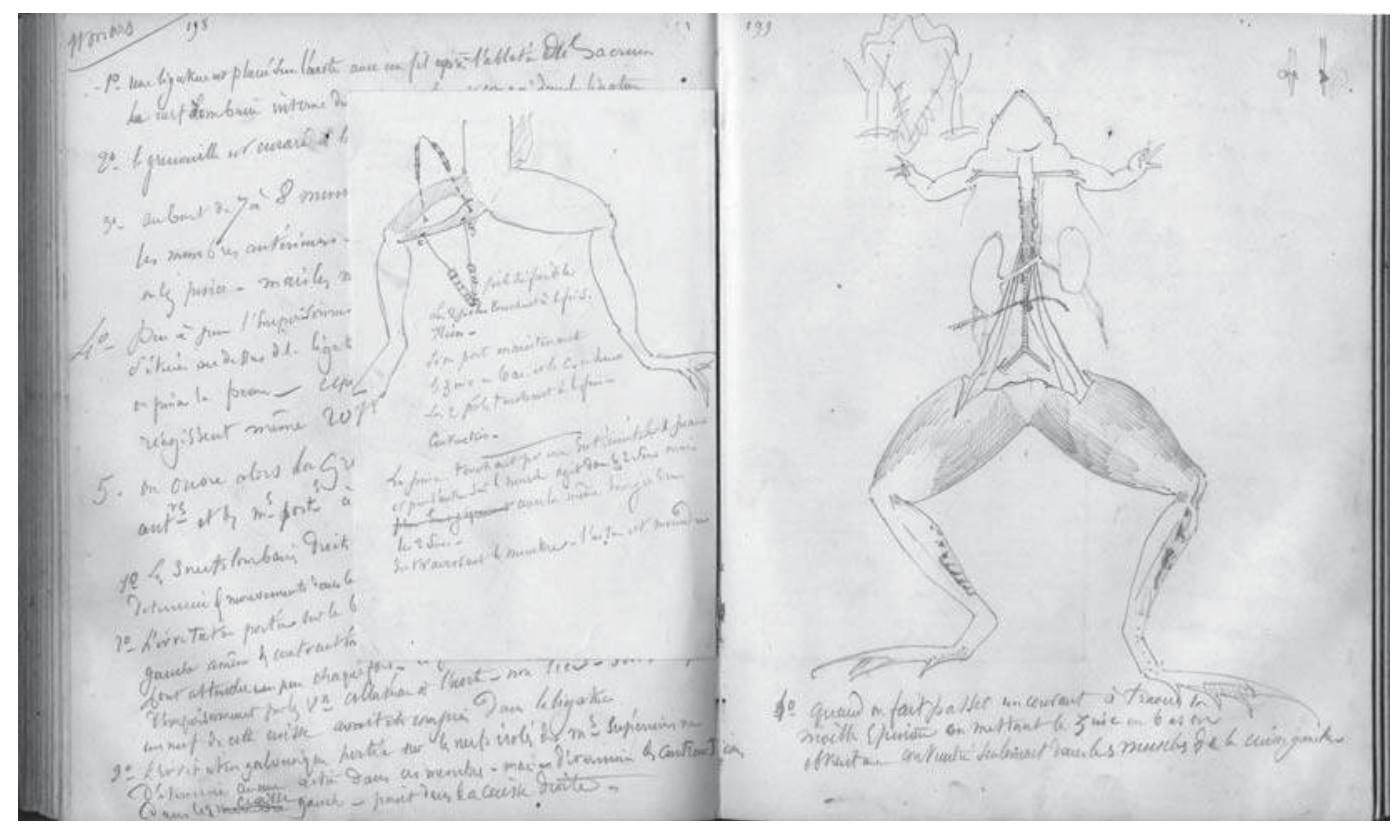

1 : Claude Bernard, Cahier 7 j, p. 198-199

(c) Fonds Claude Bernard/Collection Collège de France/IMEC

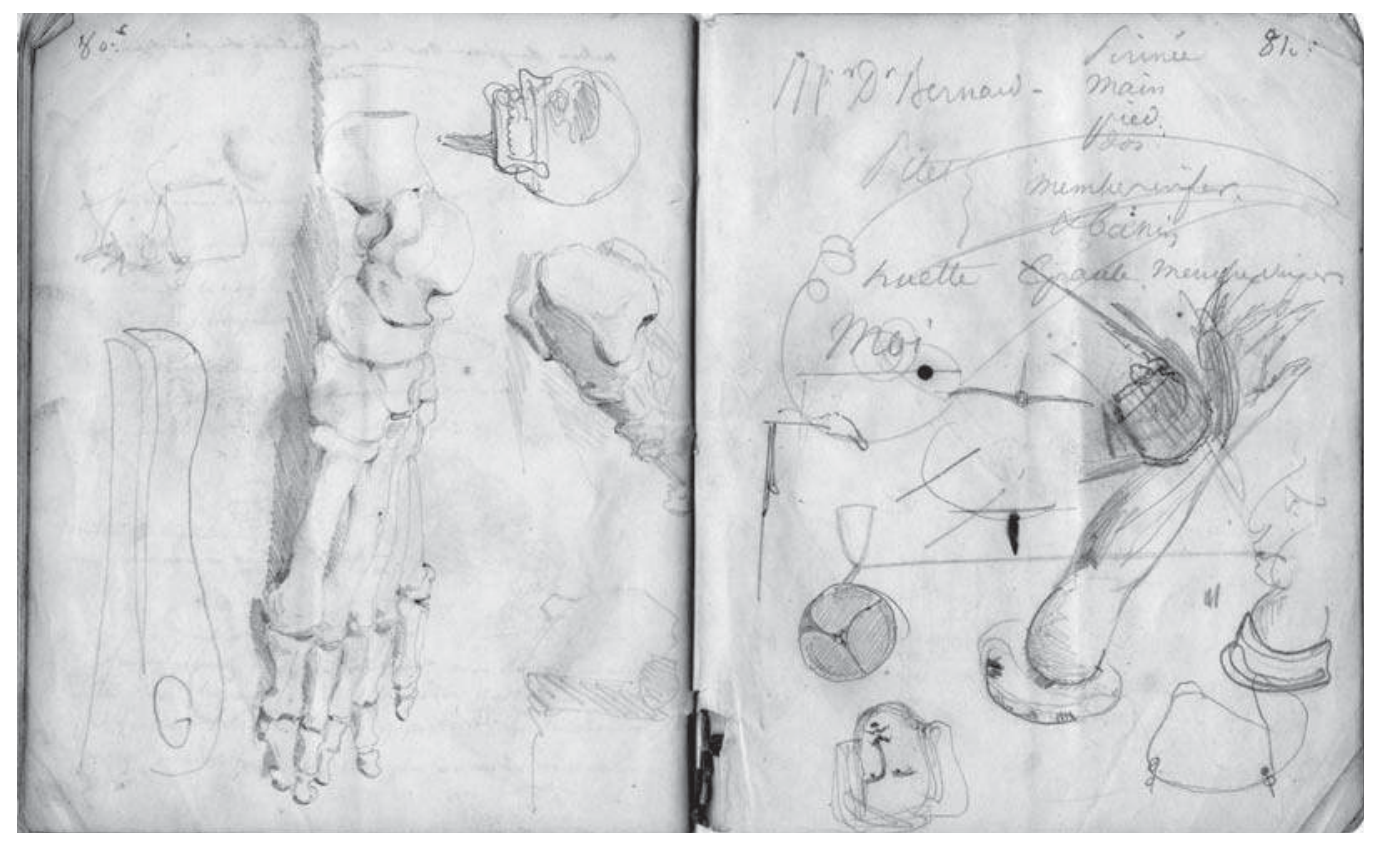

2 : Claude Bernard, Cahier 7 i, p. 80-81

(c) Fonds Claude Bernard/Collection Collège de France/IMEC 
Le cahier de laboratoire donne donc à voir une démarche qui explore beaucoup de directions. Je le dis à propos de Claude Bernard, mais le même constat vaudrait dans d'autres cas de sciences ou de problèmes scientifiques à leurs débuts. Toutes les pistes sont ouvertes, même les plus saugrenues à nos yeux - elles sont devenues invraisemblables mais au début elles ne l'étaient pas. On voit donc un chercheur aux prises avec l'inconnu, qui multiplie à dessein les protocoles d'expériences, même les plus inattendus. Claude Bernard a théorisé cette démarche avec la notion d'expérience pour voir. Et les manuscrits illustrent comment un problème scientifique se dégage petit à petit d'une espèce de gangue, faite de présupposés implicites ou de dogmes mal conçus comme tels. Les manuscrits apportent donc un éclairage irremplaçable sur le processus de création scientifique. Cet apport est d'ailleurs largement reconnu depuis longtemps.

J'ajouterai autre chose. Ce qu'on voit aussi - je l'ai vérifié sur les publications d'autres savants, mais on l'observerait certainement aussi dans les cahiers de laboratoire de Claude Bernard en approfondissant l'analyse épistémologique -, c'est qu'entre la planification initiale d'une expérience et son résultat consigné, les hypothèses peuvent changer : la réponse donnée n'est pas la réponse à la question initiale, c'est-à-dire que la question a évolué au cours de l'expérience.

J.-L. L. - Pouvez-vous dire quelques mots du projet dans le cadre duquel vous avez été amené à (re)travailler sur Claude Bernard?

C. D.- Il est effectivement intéressant de raconter l'histoire de ce projet. Cette histoire s'enracine dans la collaboration nouée par Herbert Simon, l'un des fondateurs de l'intelligence artificielle, avec l'historien des sciences Larry Holmes. Leur objectif était d'abord d'avoir, à travers les manuscrits, les publications, les interviews, etc., une idée aussi précise que possible de l'évolution d'un problème scientifique, puis, à partir de ce matériau, d'essayer, par des simulations recourant à des techniques d'intelligence artificielle, de comprendre quelle est la différence entre les processus « mécaniques » de l'intelligence artificielle et les voies et moyens par lesquels le chercheur progresse.

\section{J.-L. L. - Autrement dit d'éclairer l' invention scientifique par des méthodes d'intelligence artificielle...}

$C . D .-$ L'un des résultats très intéressants auxquels Larry Holmes et Herbert Simon sont arrivés, c'est que l'imagination fait appel à des données qui ne sont pas nécessairement consignées dans les cahiers de laboratoire ou dans les publications. C'est le problème de l'implicite, et les équipes qui collaborent au projet CYBERNARD sont en train d'être confrontées à ce problème.

J'ajouterai une chose. Ces simulations par des techniques d'intelligence artificielle ont été testées sur des matériaux d'histoire des sciences, mais elles ont aussi été conçues pour aider à la recherche médicale. Et c'est dans cette optique que Jean Bernard et Marcel Bessis ont souhaité collaborer avec Jean-Gabriel Ganascia (professeur à l'université Pierre-et-Marie-Curie) et son équipe. Parmi les résultats obtenus, je citerai la thèse de Vincent Corruble, qui a obtenu des résultats concernant les possibles déterminants familiaux ou génétiques de la lèpre ${ }^{2}$. Ces résultats ont été mis en évidence grâce à l'analyse d'un corpus de données scientifiques sur ces questions. Plusieurs années après la mort de Marcel Bessis et de Jean Bernard, il a été décidé de reprendre ce problème, toujours sur un terrain médical ou historico-scientificomédical, et nous avons lancé le projet CyBERnARD, dans lequel trois équipes coopèrent, l'équipe de JeanGabriel Ganascia pour la partie informatique, l'ITEM pour la partie génétique, et moi-même avec quelques collaborateurs pour la transcription et l'analyse épistémologique des manuscrits.

Par-delà l'intérêt intrinsèque des manuscrits de Claude Bernard sur le curare et les problèmes proprement génétiques qu'ils posent (notamment leur déchiffrement et leur mise en ordre chronologique), je voudrais souligner que, comme par un effet de miroir en retour, les questions que nous nous étions initialement posées évoluent elles aussi au fur et à mesure de la recherche. Claude Bernard cherche à définir le raisonnement expérimental. Or celui-

2. Vincent Corruble et Jean-Gabriel Ganascia, « The Discovery of the Causes of Leprosy: a Computational Analysis », AAAI, Proceedings of the Thirteenth National Conference on Artificial Intelligence, 1996, p. 731-736. 
ci n'est pas du tout formalisé, et c'est là que les choses sont intéressantes. En effet, lorsqu'on formalise selon des procédures classiques, comme l'abduction, qui est généralement considérée comme le mode de raisonnement typique de la médecine expérimentale, on s'aperçoit qu'on fige un peu les choses, et qu'on doit assouplir, modifier, élargir les procédures canoniques auxquelles on recourt habituellement.

J.-L. L. - Cela, grâce à la mise en convergence du travail sur les cahiers de laboratoire, des analyses épistémologiques...

C. D. - ... et aussi des programmes d'intelligence artificielle qui ne suivent pas nécessairement les formules quelque peu rigides héritées de la logique classique - en entendant par logique classique ce qui était plus ou moins codifié à la fin du XIXe siècle.

J.-L. L. - Les cahiers de laboratoire constituent des matériaux très riches, complexes, d'accès difficile. Est-ce que l'exploitation de ces matériaux est susceptible de modifier en profondeur un certain nombre de conceptions en histoire des sciences?

C. D. - En histoire, je n'en suis pas sûr : l'histoire des sciences à grands traits est maintenant assez largement connue. En revanche, la lecture des manuscrits sur tel ou tel problème particulier enrichit considérablement l'épistémologie du travail scientifique. Les manuscrits sont le laboratoire du chercheur, mais ils sont aussi un véritable laboratoire d'épistémologie.

J.-L. L. - Dans sa présentation des cahiers de laboratoire de Pasteur, Claire Salomon-Bayet emploie à propos du savant l'expression "Janus bifrons ${ }^{3}$ ": le savant a deux faces, il y a celui qui fait la science et celui qui la transmet. Et elle reprend dans la même présentation l'expression "penser avec les mains" utilisée par Jean-Paul Gaudillière pour qualifier les recherches de Jacques Monod, François Jacob et Jean-Pierre Changeux sur les protéines allostériques ${ }^{4}$. Pouvez-vous commenter ces deux expressions?
C.D. - Janus bifrons : je trouve que c'est un excellent point de départ. «Faire la science »-c'est précisément ce qu'on voit dans les manuscrits -, c'est imaginer des hypothèses et les tester, c'est-à-dire imaginer des situations expérimentales par lesquelles on pose des questions, enregistrer les réponses, et chercher à les interpréter en fabriquant de nouvelles hypothèses et en produisant de nouveaux résultats. C'est le processus scientifique. Et il y a toute une temporalité spécifique de ce processus d'expérimentation animale. Il y a l'échelle de la journée, il y a même l'échelle des heures : à telle heure, je fais telle expérience. Et plus tard, à telle heure... Ou alors je mets en route un lapin, et puis je mets en route une grenouille, je lui fais ceci, je vais voir ce qu'il en est à telle heure, puis je fais autre chose sur tel animal, puis je vais voir ce qu'il en est à telle heure, etc. Cette temporalité laisse sa trace dans les cahiers de laboratoire.

«Transmettre » ne se déroule pas vraiment dans la même temporalité, même si c'est généralement le même qui fait la science et qui la transmet. Il y a plusieurs échelles temporelles dans la transmission. En outre, transmettre, exposer veut dire aussi, nécessairement, figer. On s'en rend bien compte lorsqu'on prend le regard rétrospectif de la longue durée en lisant cent cinquante ans plus tard les formulations que Claude Bernard a réussi, si je puis dire, à arracher à la nature au milieu des années 1840 , et lorsqu'on les compare aux textes publiés et aux Leçons du Collège de France. On prend ainsi conscience du caractère hétérogène du processus de transmission de la science.

Toujours à propos du rapport entre la recherche telle qu'elle se fait et telle qu'elle se transmet, je voudrais citer quelque chose qui me tient à cœur. Lorsque Claude Bernard s'est exprimé pour la première fois comme professeur au Collège de France, il a dit à peu près la chose suivante : la médecine expérimentale que je suis

3. Claire Salomon-Bayet, «Introduction », dans Françoise Balibar et Marie-Laure Prévost (dir.), Pasteur. Cahiers d'un savant, Paris, CNRS Éditions, Bibliothèque nationale de France, Zulma, coll. « Manuscrits », 1995, p. 5-31.

4. Jean-Paul Gaudillière, « Carnets de laboratoire et traces de la production scientifique : de nouveaux objets pour l'histoire ? », ibid., p. 223-238. Voir aussi Claude Debru, L'Esprit des protéines, Paris, Hermann, 1983. 
chargé d'enseigner ici n'existe pas5. Cette déclaration me frappe beaucoup, et venant d'un scientifique de ce calibre, elle doit nous rendre extrêmement modestes les uns et les autres, tant les scientifiques que les historiens. L'affirmation de Claude Bernard est peut-être à mes yeux la manière la plus profondément intelligente d'appréhender la nature de la recherche scientifique.

J.-L. L. - Peut-on dire que l'approche génétique des travaux de Claude Bernard à travers ses cahiers de laboratoire confirme le jugement qu'il porte sur son enseignement à venir et aiguise notre regard rétrospectif sur la manière dont il a réellement fait naître la médecine expérimentale?

C. D. - Réellement fait naître... Il n'a pas été le seul, mais il en a été le porte-drapeau, le théoricien français. Il avait beaucoup d'imagination mais il n'avait pas toujours les instruments dont il aurait eu besoin. La situation française était à cet égard évidemment inférieure à l'énorme développement de la physiologie expérimentale en Allemagne...

J.-L. L. - C'est vrai. J'ai été très frappé dans les cahiers par le caractère relativement primitif des instruments utilisés par Claude Bernard et ses collaborateurs - et par l'ingéniosité de leur utilisation.

C. D. - Oui, Claude Bernard est un artisan et un poète. C'est ce mélange des deux qualités qui est fascinant dans les carnets de laboratoire.

En quel sens peut-on dire que Claude Bernard a créé la médecine expérimentale ? D'abord, même si elles étaient prises dans une gangue qui n'a pas résisté à l'épreuve du temps, on lui doit un nombre important de vraies découvertes. Il faut rendre à ce point hommage à Mirko Grmek, à sa thèse sur les recherches toxicologiques de Claude Bernard, tant sur l'empoisonnement par le monoxyde de carbone que sur le curare et les poisons de flèche 6 ...

J.-L. L. - . . et aussi à son immense travail d' inventaire et de classement des manuscrits ${ }^{7}$ dont la démarche, dès les années soixante, et indépendamment des premiers travaux de Louis Hay, est en phase avec la critique génétique naissante.
C.D. - En second lieu, on doit à Claude Bernard l'existence de la médecine expérimentale comme discipline enseignée en France : après lui, il y a eu des chaires de médecine expérimentale dans les universités. Elles n'ont été supprimées qu'il y a vingt ou trente ans à la suite d'une décision administrative malheureuse.

J.-L. L. - Abordons un instant l'époque présente. Que seraient à vos yeux les équivalents actuels des cahiers de laboratoire? De quels matériaux disposera-t-on dans vingt ou trente ans pour mener à bien le travail d'exploration épistémologique rendu possible pour nous par les cahiers de laboratoire de Claude Bernard?

C. D. - Ces cahiers de laboratoire, ils existent toujours. Dans l'ouvrage consacré à Pasteur dont nous parlions tout à l'heure, il y a une étude, par Jean-Paul Gaudillière, des cahiers de laboratoire de Jean-Pierre Changeux. De même, les cahiers de laboratoire étaient très présents dans le laboratoire de Michel Jouvet où, comme vous le savez, j'ai travaillé pendant plusieurs années.

Cette question m'amène à souligner à quel point il est indispensable de conserver les archives de la science. Les cahiers de laboratoire sont d'une extrême importance pour comprendre la création scientifique, et cette question sera à mon sens de plus en plus à l'ordre du jour dans les nécessaires rapprochements entre l'épistémologie et les sciences de la cognition. Les cahiers de laboratoire, mais aussi les correspondances. À l'époque de Claude Bernard, c'étaient des lettres. Maintenant, c'est le courrier électronique.

5. « Le professeur du Collège de France [...] doit avoir les yeux tournés vers l'inconnu, vers l'avenir. [...] Loin d'être achevée, la science de la vie se présentera donc à nous avec ses imperfections ; nous nous préoccuperons sans cesse, non de ce qui est fait, mais de ce qui reste à faire ; et cette direction progressive est d'autant plus importante [...] que la science dont nous nous occupons ici est plus éloignée de son entier développement », Claude Bernard, Leçons de physiologie expérimentale appliquée à la médecine, faites au Collège de France. Tome premier. Cours du semestre d' hiver 1854-1855, Paris, Baillière, 1855, p. 2-3.

6. Mirko D. Grmek, Raisonnement expérimental et recherches toxicologiques chez Claude Bernard, Genève/Paris, Droz, 1973.

7. Mirko D. Grmek, Catalogue des manuscrits de Claude Bernard, avec la bibliographie de ses travaux imprimés et des études sur son ouvre, Paris, Masson, 1967. 
Grâce à la conservation des archives, ceux qui s'attelleront à la tâche de reconstituer avec beaucoup de détails - comme le font nos collègues américains, il faut leur rendre cet hommage - la carrière de tel ou tel scientifique disposeront de données multiples, concernant non seulement la recherche scientifique, mais aussi l'organisation de la science, la politique de la science et ses aspects institutionnels, les relations internationales, etc. 11 y a là un matériau extrêmement riche qu'il faut conserver pour les générations futures parce que celles-ci devront à mon sens recevoir une éducation plus complète que celle que nous avons reçue. Pourquoi ? D'abord parce qu'un scientifique devra évidemment recevoir une formation à la fois disciplinaire et multidisciplinaire, et être capable d'intégrer ces disciplines dans l'appréhension des questions. Ensuite, parce qu'il devra avoir une perception épistémologique de ce qu'est la science et sortir du dogmatisme de l'enseignement en se donnant une perception historique de l'évolution de sa discipline, des autres disciplines, et des convergences entre disciplines.

\section{J.-L. L. - Revenons pour terminer à l' expression " penser avec les mains »...}

C. D. - En voyant travailler tel ou tel physiologiste, j'ai été souvent fasciné par ses mains. Les mains d'un physiologiste, d'un écrivain, d'un sculpteur : c'est aussi important que les mains d'un pianiste ou les mains d'un violoniste.

\section{J.-L. L. - Penser autrement qu'avec des mots...}

C. D. - J'irais plus loin, je dirais « penser avec le bout des doigts ». Ces physiologistes sont aussi des chirurgiens. Et pendant toute une période, la sensation a certainement joué un rôle important au cours du processus chirurgical. Évidemment, nous sommes entrés maintenant dans l'ère de l'imagerie, et les auxiliaires qu'elle procure sont devenus très importants en chirurgie, mais pendant toute cette époque qui a vu le développement de la médecine expérimentale, les physiologistes étaient des chirurgiens. La neurochirurgie a joué un rôle considérable dans les neurosciences, par exemple en Amérique avec Cushing et ses travaux sur les tumeurs de l'hypophyse qui ont éclairé beaucoup de questions en neuroendocrinologie. Claude Bernard a passé son temps à faire de la chirurgie!

\section{J.-L. L. - Et on en trouve la consignation...}

C.D. - ... dans ses cahiers. « Penser avec le bout des doigts », c'est avoir cette espèce de feedback entre la représentation et la sensation. Pour penser, c'est-à-dire élaborer des hypothèses, ou des représentations quelles qu'elles soient, il est nécessaire de soumettre celles-ci au test de l'expérience. Or les mains savent. Elles savent que chez tel animal, tel nerf se trouve ici, qu'à côté il y a tel autre nerf. Donc on ne peut pas penser sans les mains, on ne peut pas réaliser une expérience sans avoir cette connaissance acquise dans les mains. Donc un physiologiste est nécessairement quelqu'un qui pense avec les mains, et ce sont souvent de très belles mains.

J.-L. L. - De la main qui écrit et qui dessine à la main qui pense : n'est-ce pas un magnifique raccourci de l'enjeu de la critique génétique?

Claude Debru, Ancien élève de l'ENS, agrégé de philosophie, membre correspondant de l'Académie des sciences. Principaux ouvrages : L'Esprit des protéines (Paris, Hermann, 1983), Neurophilosophie du rêve (Paris, Hermann, 1989, deuxième édition 2006), Philosophie de l' inconnu : le vivant et la recherche (Paris, PUF, 1998), Le Possible et les biotechnologies (Paris, PUF, 2003), Georges Canguilhem : science et non-science (Paris, ENS Ulm, 2004), L'Essor des neurosciences France 1945-1975 (C. Debru, J.-G. Barbara et C. Chérici (éds), Paris, Hermann, 2007), L'Imagination et l' intuition dans les sciences (P. Buser, C. Debru et A. Kleinert (éds), Paris, Hermann, 2009).

Claude Debru, Claude.Debru@ens.fr 